\title{
Unintentional Ingestion of Foreign Bodies in the Laryngopharynx
}

\author{
Chin-Lung Kuo ${ }^{1,2,4,5,6}$, Nancy HsaoYen Chang ${ }^{3}$ \\ ${ }^{1}$ Department of Otolaryngology, HsinChu Armed Forces General Hospital, HsinChu, Taiwan, ROC \\ ${ }^{2}$ Institute of Brain Science, National Yang-Ming University, Taipei, Taiwan, ROC \\ Taipei, Taiwan, ROC \\ ${ }^{3}$ College of Natural Sciences, University of Texas at Austin, Austin, Texas, USA \\ ${ }^{4}$ Department of Otolaryngology, National Yang-Ming University School of Medicine, \\ Taipei, Taiwan, ROC \\ ${ }^{5}$ Department of Otolaryngology-Head and Neck Surgery, Taipei Veterans General Hospital \\ ${ }^{6}$ Department of Otolaryngology, Taoyuan Armed Forces General Hospital, Taoyuan, Taiwan, ROC
}

\section{INTRODUCTION}

Unintentional ingestion of foreign bodies is a common occurrence. Although accidental foreign body ingestion is rarely associated with complications, a fatal event can cause disastrous consequences, which require surgical exploration. Previous research has revealed that surgical intervention rates range from $12 \%$ to $16 \% .{ }^{1,2}$ We report a case of an impacted large chicken bone in the laryngopharynx in a 66-year-old man.

\section{Case Presentation}

A 66-year-old man presented with severe neck pain on the left side and painful swallowing for 2 days. The patient had requested medical advice at a primary health care facility and was treated with acetaminophen. However, his symptoms increased in severity and were later accompanied by progressive hoarseness. A detailed medical history of the patient revealed that he had suffered an attack of choking and coughing immediately after eating chicken one day before the onset of symptoms. The author therefore conducted an endoscopic nasopharyngeal fiberoptic examination, which revealed a large chicken bone with an ovoid head impacted at the left pyriform fossa (Figure). Unilateral left vocal cord paralysis was with airway compromise was also found. Given that stridor and progressive shortness of breath were observed during the examination, the patient underwent emergency endoscopic retrieval of the chicken bone.

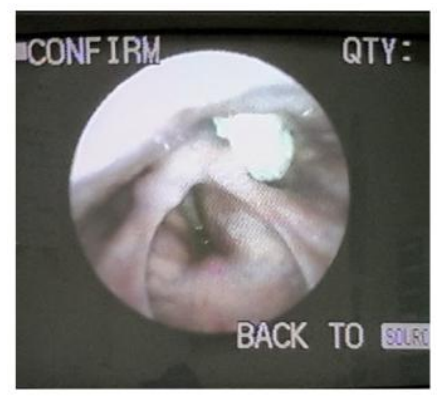

\section{DisCUSSION}

The most common types of foreign body ingested are fishbone and coin in children; and fish and chicken bones in adults. ${ }^{3,4}$ A study of 1,338 patients by Lai et.al revealed that the commonest location for a foreign body was the valleculae $(31.4 \%){ }^{4}$ On most occasions, the ingested fishbone passes uneventfully through the gastrointestinal tract within one week. ${ }^{5}$ However, ingestion of foreign body may pose a potentially lethal risk, which mandates surgical exploration. ${ }^{5}$ For patients complaining of accidental foreign body ingestion, flexible endoscopic nasopharyngoscopy may be required to detect the foreign body, regardless of plain lateral neck radiographic findings. CT scan of neck may be useful 
when plain lateral radiographs of the neck fail to detect the fish bone. A missed or delayed diagnosis can lead to respiratory distress and even complete airway obstruction or death. Clinicians and patients should be made aware of this potentially fatal situation.

\section{REFERENCES}

[1] Palta R, Sahota A, Bemarki A, Salama P, Simpson N, Laine L. Foreign-body ingestion: characteristics and outcomes in a lower socioeconomic population with predominantly intentional ingestion. Gastrointestinal endoscopy 2009; 69:426-433.

[2] Weiland ST, Schurr MJ. Conservative management of ingested foreign bodies. Journal of gastrointestinal surgery : official journal of the Society for Surgery of the Alimentary Tract 2002; 6:496-500.

[3] Nandi P, Ong GB. Foreign body in the oesophagus: review of 2394 cases. The British journal of surgery 1978; 65:5-9.

[4] Lai AT, Chow TL, Lee DT, Kwok SP. Risk factors predicting the development of complications after foreign body ingestion. The British journal of surgery 2003; 90:1531-1535.

[5] Bathla G, Teo LL, Dhanda S. Pictorial essay: Complications of a swallowed fish bone. The Indian journal of radiology \& imaging 2011; 21:63-68. 